\title{
Analysis of heterogeneity and anisotropy of diffusivity in the sandy facies of Opalinus Clay host rocks using multi-scale digital rock physics
}

\author{
TAO YUAN ${ }^{1}$, YUANKAI YANG ${ }^{2}$, GUIDO DEISSMANN ${ }^{2}$ \\ AND CORNELIUS FISCHER ${ }^{3}$ \\ ${ }^{1}$ Helmholtz-Zentrum Dresden-Rossendorf (HZDR), Institute of \\ Resource Ecology \\ ${ }^{2}$ Forschungszentrum Jülich GmbH \\ ${ }^{3}$ Helmholtz-Zentrum Dresden-Rossendorf (HZDR), Institute of \\ Resource Ecology, Reactive Transport Department \\ Presenting Author: t.yuan@hzdr.de
}

The effective diffusivity is a key parameter in numerical tools required for the simulation of radionuclide migration in lowpermeable rocks. Potential host rocks for deep geological repositories for nuclear waste such as the Opalinus Clay (OPA) exhibit pore network heterogeneities at the nanometer to micrometer scale. In the sandy facies of OPA, this pore network is critically modified due to compositional variability and owing to diagenetic reaction products, e.g., carbonate minerals. Such spatial variability is responsible for heterogeneous and anisotropic diffusion patterns contrary to the commonly assumed homogeneous conditions in the shaly facies. At the continuum scale, the representative elementary volume (REV) is a fundamental parameter for the quantification of the effective diffusivity. Therefore, meaningful modeling of heterogeneous and anisotropic diffusion in the sandy facies of OPA at the continuum scale requires an accurate estimation of the REV.

Here, we first utilize digital rock physics and propose an upscaling workflow that integrates transport simulations at both the nanometer-scale and the micrometer-scale to estimate the effective diffusivity of radionuclides in the sandy facies of OPA. In the proposed upscaling workflow, the lattice Boltzmann method (LBM) is used to solve the Poisson-Nernst-Planck (PNP) equation at the nanometer-scale [1]. The nanometer-scale results are then used as input parameters in the micrometer-scale model for diffusive transport calculations. At the micrometer-scale, the three-dimensional (3D) diffusion-sorption equation is numerically solved by a previously developed numerical simulator [2]. The diffusivity is calculated using the proposed upscaling approach, which is validated with published experimental data that confirm the general applicability of the models. Next, we determine the REV for diffusivity by analyzing the calculated diffusivity of the selected regions of interest (ROIs) as a function of the length scale [3]. The determined REV provides critical insight into the heterogeneity and anisotropy of the diffusivity in the sandy facies of OPA, which contributes to enhanced predictability of radionuclide migration.

[1] Yang et al. (2019) Cem. Concr. Compos. 96, 55-65.

[2] Yuan et al. (2019) Water, 11 (10).

[3] Houben et al. (2014) Mar. Pet. Geol. 49, 143-161. 